\title{
5G MIMO Conformal Microstrip Antenna Design
}

\author{
Qian Wang, ${ }^{1}$ Ning Mu, ${ }^{1}$ LingLi Wang, ${ }^{1}$ Safieddin Safavi-Naeini, ${ }^{2}$ and JingPing Liu ${ }^{1}$ \\ ${ }^{1}$ School of Electronic and Optical Engineering, Nanjing University of Science and Technology, Nanjing, Jiangsu 210094, China \\ ${ }^{2}$ Electrical and Computer Engineering Department, University of Waterloo, Waterloo, ON, Canada N2L 3G1 \\ Correspondence should be addressed to JingPing Liu; liujingpin2002@aliyun.com
}

Received 14 June 2017; Revised 29 October 2017; Accepted 23 November 2017; Published 17 December 2017

Academic Editor: Pai-Yen Chen

Copyright (C) 2017 Qian Wang et al. This is an open access article distributed under the Creative Commons Attribution License, which permits unrestricted use, distribution, and reproduction in any medium, provided the original work is properly cited.

\begin{abstract}
With the development of wireless communication technology, 5G will develop into a new generation of wireless mobile communication systems. MIMO (multiple-input multiple-output) technology is expected to be one of the key technologies in the field of $5 \mathrm{G}$ wireless communications. In this paper, 4 pairs of microstrip MIMO conformal antennas of $35 \mathrm{GHz}$ have been designed. Eight-element microstrip Taylor antenna array with series-feeding not only achieves the deviation of the main lobe of the pattern but also increases the bandwidth of the antenna array and reduces sidelobe. MIMO antennas have been fabricated and measured. Measurement results match the simulation results well. The return loss of the antenna at $35 \mathrm{GHz}$ is better than $20 \mathrm{~dB}$, the first sidelobe level is $-16 \mathrm{~dB}$, and the angle between the main lobe and the plane of array is $60^{\circ}$.
\end{abstract}

\section{Introduction}

Multiple-input multiple-output (MIMO) technology is originated from wireless communication antenna diversity technology and intelligent antenna technology. It is a combination of multiple-input single-output (MISO) and single-input multiple-output (SIMO) and therefore has the advantages and characteristics of the two $[1,2]$. The MIMO system is equipped with multiple antennas at the transmitter and the receiver. It can improve the quality of wireless communication and the rate of data exponentially without increasing the bandwidth and transmitted power $[3,4]$. Multiantenna system is an important part of MIMO technology. MIMO wireless system is not only affected by the multipath characteristics of the wireless communication channel but also depends on the design and layout of the multiantenna system. The research of MIMO multiantenna design mainly includes the form of antenna element, the layout of multiple antennas, and the mutual coupling analysis. At present, the research of MIMO multiantenna is focused on the exploration of low cost and high performance designs of antenna and layout [5-7].

Antennas are usually placed on the surface of the carrier to achieve the desired electromagnetic performance. To this end, the conformal antenna was designed [8]. Conformal antenna can be designed on the surface of the carrier, which will not damage the mechanical structure of the carrier and can save space [9-11]. It can be placed anywhere on the surface of the carrier. Conformal antennas are usually microstrip antenna, stripline antenna, or crack antenna. The microstrip antenna has many advantages such as low profile, small size, light weight, and ease to integrate with other carriers. It is therefore more suitable for conformal antenna [12, 13]. In addition, the millimeter-wave band has attracted a lot of attention due to the advent of 5G technology and its inherent characteristics, such as short wavelength, wide frequency band, and propagation characteristics in the fog, snow, and dust environment [14-16]. Therefore, there has been extensive research on millimeter-wave microstrip antenna.

This paper presents the design of a MIMO conformal antenna for $5 \mathrm{G}$. The frequency is $35 \mathrm{GHz}$, the carrier of conformal is a cylinder, and the angle between the main lobe of pattern and the carrier axis is $60^{\circ}$. The sidelobe characteristics of the antenna significantly affect the interference of the system and the suppression of the clutter. The antenna designed in this paper requires the first sidelobe level to be about $-18 \mathrm{~dB}$. In view of this characteristic, a seriesfed standing wave antenna array with Taylor distribution is designed. Considering the influence of coupling, 4 pairs of antennas are designed. The results of the research are well suited for the 5 G MIMO communication. 


\section{5G MIMO Conformal Antenna Design}

2.1. Radiation Elements Design. The first part of the MIMO conformal antenna design is the radiation elements. The design uses microstrip patch antenna as radiation elements. There are two main steps in the rectangular microstrip antennas design. The first step is theoretical analysis, and the second step is simulation and optimization.

Firstly, we choose dielectric substrate. For microstrip circuit, the loss of the microstrip is very large in the millimeterwave band. The loss can be divided into dielectric loss, conductor loss, and radiation loss [17]. Substrates with low loss tangent dielectric are usually chosen to reduce the dielectric loss. When the dielectric constant is low, the total loss of the microstrip would not change with the characteristic impedance. On the contrary, when the substrate has high dielectric constant, the loss of the microstrip will change rapidly with the characteristic impedance. Thicker substrate will increase radiation losses and the surface wave is more serious. A smaller height is more effective in suppressing the higher mode and reducing the radiation loss. Additionally, the thinner substrate with good flexibility is good for conformal antenna [18]. Taking these factors into consideration, we use RT/duroid5880 $\left(\varepsilon_{r}=2.2, \tan \delta=0.0009\right)$ as substrate, and the height of the dielectric substrate is $0.5 \mathrm{~mm}$.

Next the width $W$ of the patch elements is determined. Directivity factor of microstrip antenna, radiation resistance, and other characteristics will vary with the change of $W$. These characteristics directly affect the frequency bandwidth and radiation efficiency of the antenna. In order to get the desired frequency bandwidth and radiation efficiency, the choice of $W$ is particularly important. The size of the width should meet the following requirement [18]:

$$
W \leq \frac{c}{2 f_{r}}\left(\frac{\varepsilon_{r}+1}{2}\right)^{-1 / 2},
$$

where $c$ is speed of light and $f_{r}$ is the resonant frequency. In this design, $f_{r}$ is $35 \mathrm{GHz}$.

The next step is to determine the length $L$ of the patch elements. The size of unit length $L$ is determined by the effective dielectric constant and the operating frequency. Effective dielectric constant of substrate is defined as $\varepsilon_{e}$, which is given by the following [18]:

$$
\varepsilon_{e}=\frac{1}{2}\left[\varepsilon_{r}+1+\left(\varepsilon_{r}-1\right)\left(1+\frac{12 h}{W}\right)^{-1 / 2}\right] .
$$

The length $L$ of the rectangular microstrip patch antenna is approximately $\lambda_{g} / 2$ and is given by the following [18]:

$$
\begin{aligned}
\Delta l & =0.412 \frac{\left(\varepsilon_{e}+0.3\right)(W / h+0.264)}{\left(\varepsilon_{e}-0.258\right)(W / h+0.8)} h \\
L & =\frac{c}{2 f_{r} \sqrt{\varepsilon_{e}}}-2 \Delta l .
\end{aligned}
$$

From (1)-(3), $W$ is chosen to be $3.38 \mathrm{~mm}$ and $L$ is $2.8 \mathrm{~mm}$.

There are three main methods to feed the rectangular microstrip patch. Microstrip line feeding is usually used to

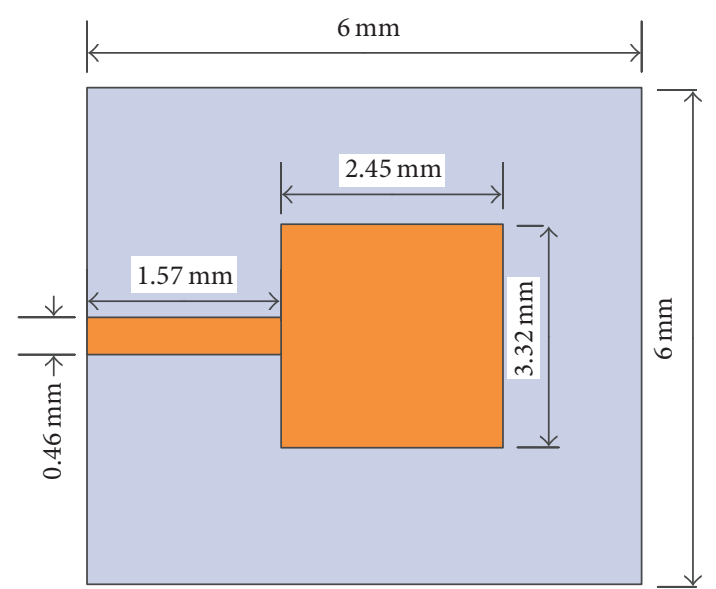

Figure 1: The model of rectangular microstrip patch antenna.

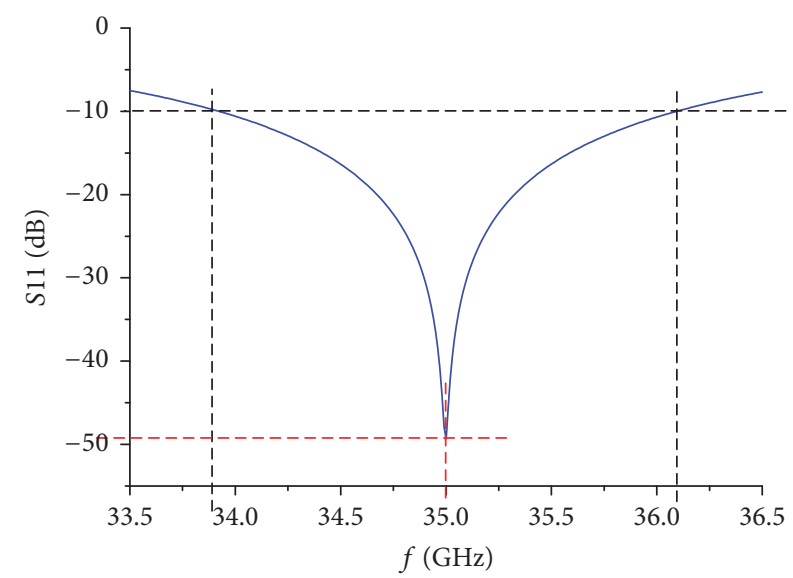

FIGURE 2: S-parameter of rectangular microstrip patch antenna.

design array elements, coaxial feeding is usually used for single microstrip antenna, and the electromagnetic coupling feeding is usually used in the microstrip antenna of double structure [18]. Microstrip patch element designed here is a radiation element in the antenna array, so microstrip is chosen as feeding method.

The rectangular microstrip antenna element is shown in Figure 1. $W$ and $L$ of the patch element are adjusted during the simulation process. The size of $L$ mainly affects the resonant frequency, and $W$ mainly affects impedance matching. Through simulation and optimization, we get that $W=3.32 \mathrm{~mm}$ and $L=2.45 \mathrm{~mm}$, the width of feed line $w=0.46 \mathrm{~mm}$, and the center of the feed line is midpoint of $W$.

All simulations are performed using HFSS in the following. Figure 2 is $S$-parameter of rectangular microstrip patch antenna. It can be seen from the figure that return loss has reached $-49 \mathrm{~dB}$ at $35 \mathrm{GHz}$. The relative bandwidth for $\left|S_{11}\right|<$ $-10 \mathrm{~dB}$ can be found from the figure to be $6.6 \%$.

In this design, the line width of the microstrip line is $0.46 \mathrm{~mm}$, and the characteristic impedance is $50 \Omega$. Therefore, the input impedance of the patch element needs to be close to $50 \Omega$. Figure 3 is the input impedance of rectangular microstrip patch antenna. From the figure, the input 


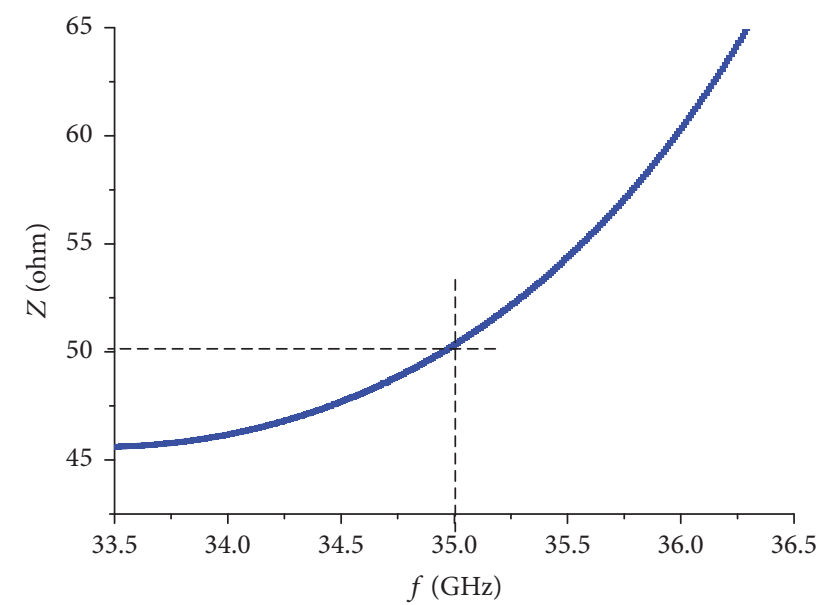

FIGURE 3: The input impedance of rectangular microstrip patch antenna.

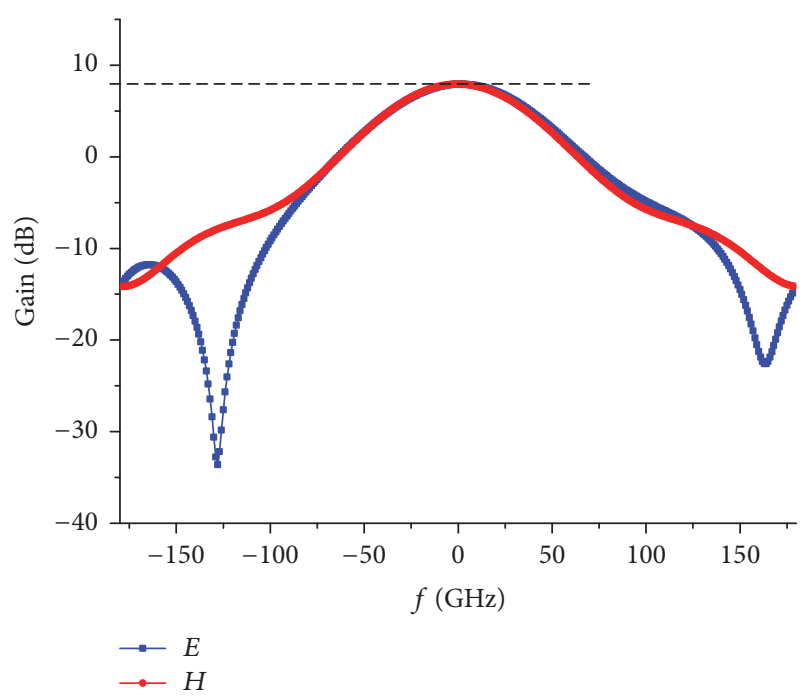

FIGURE 4: The gain of rectangular microstrip patch antenna.

impedance is about $50.34 \Omega$, which matches well to the characteristic impedance of the microstrip line.

Figure 4 is the gain of rectangular microstrip patch antenna, which shows that the maximum gain of the patch element is $7.93 \mathrm{~dB}$, the $3 \mathrm{~dB}$ lobe-width of $E$-Plane is $76^{\circ}$, and the $3 \mathrm{~dB}$ lobe-width of $H$-plane is $73^{\circ}$. The pattern of the patch is consistent with the theory and the main lobe-width of $E$ plane is slightly larger than the $H$-plane.

The frequency band width of the microstrip antenna is not enough for the whole system. Therefore, in the following, we will analyze the array antenna to meet the requirements of the frequency bandwidth.

2.2. Theoretical Analysis of Series-Fed Array. The second part of MIMO conformal antenna design is the microstrip seriesfed array. To get high gain, low sidelobe, beam scanning, and beam control, we need to use the discrete radiating element to form the array according to the appropriate excitation and distance. In this paper, the requirements of the microstrip

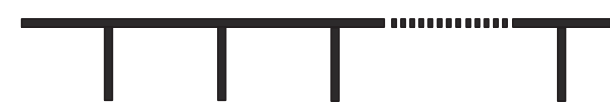

FIGURE 5: The radiating element connected with a fine line to realize the feeding.

array are as follows: the gain is $10 \mathrm{~dB}$, the angle between the main lobe and plane of array is not less than $10 \%\left(\left|S_{11}\right|<\right.$ $-10 \mathrm{~dB}$ ), and the first sidelobe level is about $-18 \mathrm{~dB}$. The design of microstrip is divided into three steps. The first step is to select the feed method of the linear array, the second step is to realize the offset of the main lobe, and third step is to reduce the first sidelobe level.

For the antenna array, the feeding method can be formed with parallel feed and series feed or the combination of the two $[18,19]$. In this paper, we use series feed, as shown in Figure 5. The radiating elements are connected through a microstrip line, and the end is open circuit. The first radiating element is fed by a coaxial line. In order to avoid the influence of the microstrip on the antenna radiation, it is necessary to make it as thin as possible.

The second step is to realize the offset of the main lobe of the antenna pattern. There are typically series-fed traveling-wave array and the series-fed standing wave array. For series-fed traveling-wave array, the distance between radiating elements can be adjusted to achieve the offset of the main lobe. However, in the design of standing wave array with series-feeding, as long as the input impedance of the last radiating element of the array is designed to be consistent with the characteristic impedance of the microstrip line, it can also play a role in impedance matching to achieve the effect of traveling-wave array. This design uses a series of standing wave array, as shown in Figure 5. The advantage of this array is that it does not require the addition of terminal load. In the design of the radiating element, the input impedance of the radiating element is designed near the characteristic impedance of the microstrip. Therefore, the radiating element can be regarded as the matched load. We can change the phase relationship between elements by adjusting the distance between them in order to realize the arbitrary beam direction and achieve the effect of the main lobe [20].

For the design of a series-fed traveling-wave array, assuming that the main lobe angle from the end fire direction is $\theta$, the relationship between the main lobe direction angle and the radiating element spacing is as follows:

$$
\cos \theta=\frac{\lambda}{\lambda_{g}}-\frac{\lambda}{S}
$$

where $S$ is the distance between the radiating elements and $\lambda_{g}$ is the effective wavelength in the medium. When the distance $S<\lambda_{g}$, the main lobe biases feed; otherwise, it biases load. Element spacing $S$ is an important parameter influencing the radiation characteristics of an antenna array. In order to avoid grating lobes, radiating element spacing $S$ needed to meet the following formula:

$$
S<\frac{\lambda_{0}}{1+\left|\cos \theta_{m}\right|}
$$


TABLE 1: Normalized current value of eight-element Taylor array.

\begin{tabular}{lcccccccc}
\hline Unit number & 1 & 2 & 3 & 4 & 5 & 6 & 7 & 8 \\
\hline Normalized current & 0.6 & 0.63 & 0.83 & 1 & 1 & 0.83 & 0.63 & 0.6 \\
\hline
\end{tabular}

TABLE 2: $S_{12}$ of eight-element Taylor array.

\begin{tabular}{lccc}
\hline Unit number & 2 & 3 & 4 \\
\hline$S_{12}$ & -3.1792 & -2.8252 & -2.2721 \\
\hline
\end{tabular}

Formulas (4) and (5) are for traveling wave. In this paper, the design of the standing wave array can also use these two formulas. The distance between the radiating elements of the standing wave array obtained by the above two formulas is $4.41 \mathrm{~mm}$.

The third step is to reduce the sidelobe level. The antenna design is based on 8-element linear array. Because of the need to achieve the main lobe deviation, the distance between the radiating elements is consistent. The sidelobe amplitude can be reduced by controlling the current. The current amplitude distribution design is based on the Taylor distribution [21, 22]. In the comprehensive design of the Taylor, it is necessary to determine the ratio $R$ of the main lobe level to the sidelobe level. The value of $A$ is calculated by $R$. Under the guarantee of $\bar{n} \geq 2 A^{2}+1 / 2$, selecting appropriate $\bar{n}$ (the value of $\bar{n}$ increases, the value of $\sigma$ decreases, and the lobewidth narrows down). The value of $\bar{n}$ should not be too large; otherwise, the amplitude distribution of the current will change dramatically. After selecting $\bar{n}$, beam broadening factor $\sigma$ and current amplitude distribution of each radiating element can be calculated. Ratio of the main lobe level to the sidelobe level is $R=-18 \mathrm{~dB}, \bar{n}=4$. The normalized current values of all levels are shown in Table 1.

There are two methods to change the current amplitude distribution. The first one is $\lambda / 4$ impedance transformer, and the second is the patch width distribution method. Due to the relatively small spacing of the radiating elements, the $\lambda / 4$ section cannot be added, so the patch width distribution method is used to change the current amplitude distribution. In fact, the change of current amplitude distribution can be realized by changing the radiation admittance of the element. Firstly, $S_{12}$ of eight-element Taylor array at all levels should be calculated according to the current distribution. Feeding in this paper is from the center to both ends of the array. Therefore, according to the symmetry, only half of the array needs to be considered where calculating $S_{12}$. The calculated results are shown in Table 2. According to these values, the width of each radiating element can be adjusted, and the appropriate size can be found to satisfy the current amplitude distribution through simulation and optimization.

2.3. Simulation and Analysis of Microstrip Series-Fed Linear Array. The model of rectangular array with uniform distribution of one-end feeding is shown in Figure 6. XOY plane is the plane of the array. The rectangular microstrip patches with the same shape are used to design the array element. The spacing between patches is the same. First, we adjust the unit spacing to meet the main direction deflection of the beams of the microstrip series-fed linear array. Then, the array is connected to the external $50 \mathrm{ohm}$ coaxial line. Because the impedance of the whole array is not matched to the $50 \mathrm{ohm}$ coaxial line, an impedance transforming section should be added at the front of the array to match the impedance. The length of the section is $\lambda_{g} / 4$. After optimization, the distance between the radiating element and the radiating element is $4.19 \mathrm{~mm}$.

The $S$-parameter of a rectangular array with uniform distribution of one-end feeding is shown in Figure 7. It can be seen from the figure that, at the center frequency $35 \mathrm{GHz}$, the return loss is $-27.7 \mathrm{~dB}$. The relative bandwidth $\left|S_{11}\right|<-10 \mathrm{~dB}$ can be found from the figure to be $26.14 \%$.

The impedance of a rectangular array with uniform distribution of one-end feeding is shown in Figure 8. It can be seen that the antenna is well matched at $48.8 \Omega$.

The $E$-plane gain is shown in Figure 9. The maximum gain is $13.79 \mathrm{~dB}$. The first sidelobe level is $-13.2 \mathrm{~dB}$. The main lobe deflection offset is achieved on $E$-plane, the angle is about $60^{\circ}$, and the $3 \mathrm{~dB}$ lobe-width in the $E$-plane is $16.8^{\circ}$.

The first sidelobe level is higher, which cannot meet the design requirements. We need to find the appropriate spacing between elements to meet the main beam deflection. Then the current distribution is designed to reduce the sidelobe level.

In this paper, the Taylor current distribution is used to reduce the sidelobe level. Taylor distribution is usually used in the form of intermediate feed. The spacing between radiation units usually takes one wavelength. As the design needs to achieve main beam offset, the spacing is no longer a wavelength. The feed position is required to be transferred to the center of the array, and the form needs to be adjusted. For an array which makes main beam deviation through changing the spacing between the radiation units, we in fact change the current phase difference between the radiating elements and then the main beam is offset. Considering the current phase difference for the whole array, add serpentine at one side of the array to adjust the phase difference and, at another side of the serpentine, the phase difference of 180 degrees should be added at the beginning of the unit. Then, adjust the current phase difference between the left and right arrays.

From the simulation results, we know that the unit spacing which can satisfy the main beam offset is $4.19 \mathrm{~mm}$. The next step is to transfer the feed position to the center of the array, and add serpentine at one side of the array, determine the length of serpentine through the simulation. Before the design of Taylor matrix, we need to design a uniformly distributed rectangular array with intermediate feed to decide the length of serpentine. The design is shown in Figure 10; XOY plane is the model plane.

The rectangular microstrip patches with the same shape are used to design the array element. The spacing between antennas is the same. The feed structure of this array is in the middle of the array and is connected to a $50 \Omega$ coaxial line. The design process is similar to that of a rectangular array with uniform distribution at one end of the feed. The design is divided into two parts. As can be seen from Figure 10, the left end of the feed is the same as the uniform distribution of one-end feeding. In the right end, serpentine lines are added to realize phase array progression, so as to realize the beam 


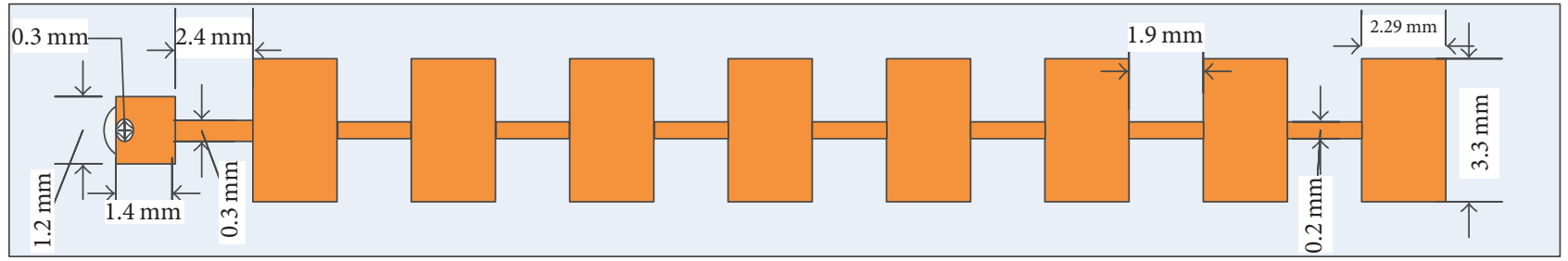

FIGURE 6: Model of rectangular array with uniform distribution of one-end feeding.

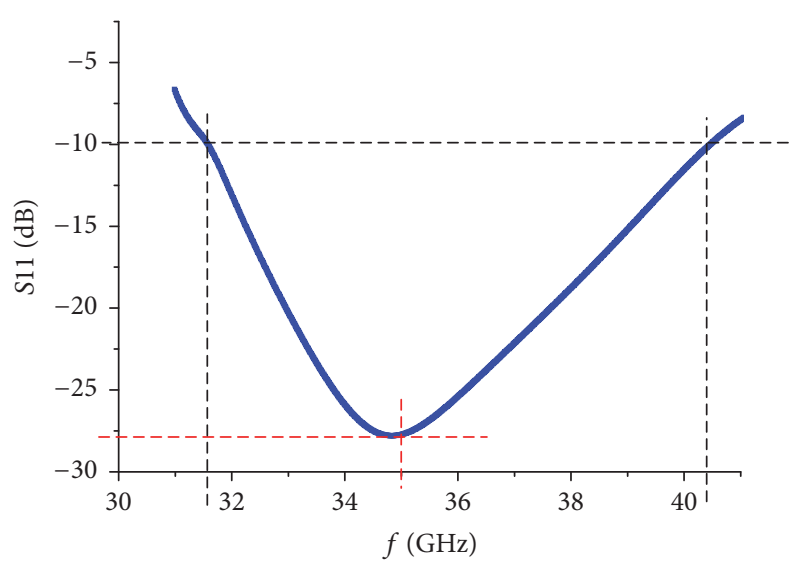

FIGURE 7: $S$-parameter diagram of a rectangular array with uniform distribution of one-end feeding.

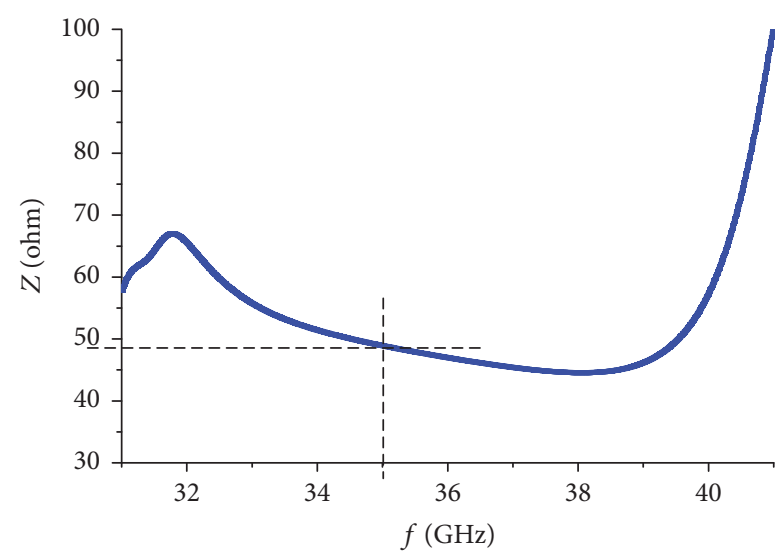

FIGURE 8: Impedance of a rectangular array with uniform distribution of one-end feeding.

deviation. After the simulation, the length of the serpentine is found to be $6.51 \mathrm{~mm}$.

The $S$-parameter of a rectangular array with uniform distribution and intermediate feeding is shown in Figure 11. It can be seen from the plot that, at the center frequency of $35 \mathrm{GHz}$, the return loss is up to $-31.87 \mathrm{~dB}$. The relative bandwidth $\left|S_{11}\right|<-10 \mathrm{~dB}$ can be found to be $21.7 \%$.

The impedance is shown in Figure 12. It can be seen from the figure that the antenna is well matched at $51.5 \Omega$. The gain graph of $E$-plane is shown in Figure 13. It can be seen from the figure that the maximum gain is $13.36 \mathrm{~dB}$, the first sidelobe level is $-13.7 \mathrm{~dB}$, the main beam deflection offset is achieved

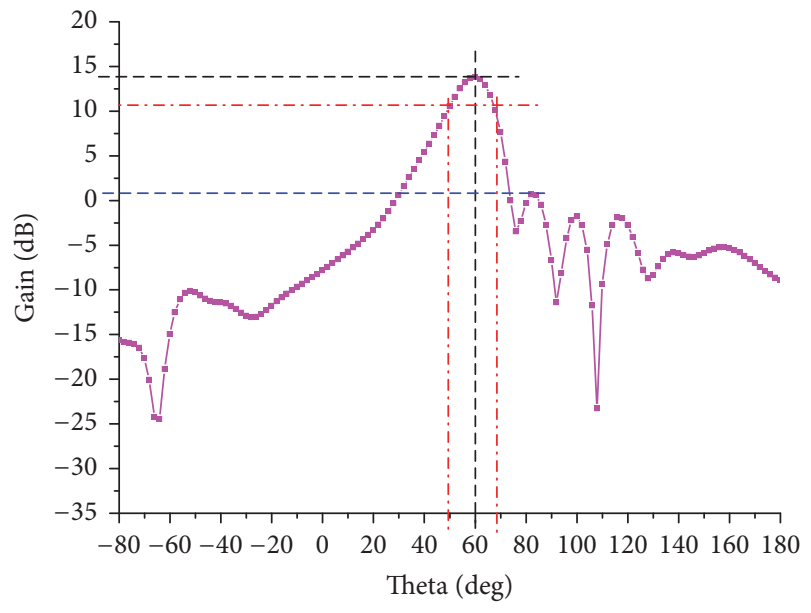

FIGURE 9: The gain of E-plane of a rectangular array with uniform distribution of one-end feeding.

on $E$-plane, the angle is about $62^{\circ}$, and the $3 \mathrm{~dB}$ lobe-width in the $E$-plane is $18^{\circ}$.

The designed array has satisfied the requirement of the main beam offset, but the sidelobe level is still too high. The feeding position is in the middle of the array, which satisfies the design of Taylor distribution. The design of Taylor distribution is carried out based on this array. The model diagram is shown in Figure 14; XOY plane is the plane of the array.

The form of the array is the same as that of the middle feed, and there is a difference in the size of the array. The size of the array element is designed according to Taylor current distribution regulation $S_{12}$ of the elements at all levels which can be obtained from Table 2. By adjusting the radiation edge size of each element, the radiation admittance of each element can be changed, and the corresponding value of the radiation edge size can be obtained.

After simulation and optimization, from the feed point to the right, the sizes of radiation side are $W_{1}=3.7 \mathrm{~mm}, W_{2}=$ $3.4 \mathrm{~mm}, W_{3}=4.1 \mathrm{~mm}, W_{4}=3.2 \mathrm{~mm}$.

The $S$-parameter diagram of a rectangular array with Taylor distribution and intermediate feed is shown in Figure 15. It can be seen from the figure that at the center frequency the return loss is very high $(21.2 \mathrm{~dB}$ at $35 \mathrm{GHz})$. The relative bandwidth $\left|S_{11}\right|<-10 \mathrm{~dB}$ can be found from the picture to be $11.6 \%$.

The impedance is shown in Figure 16. It can be seen that the antenna is well matched at $50.1 \Omega$. The gain graph of $E$ plane is shown in Figure 17. It can be seen from the figure 


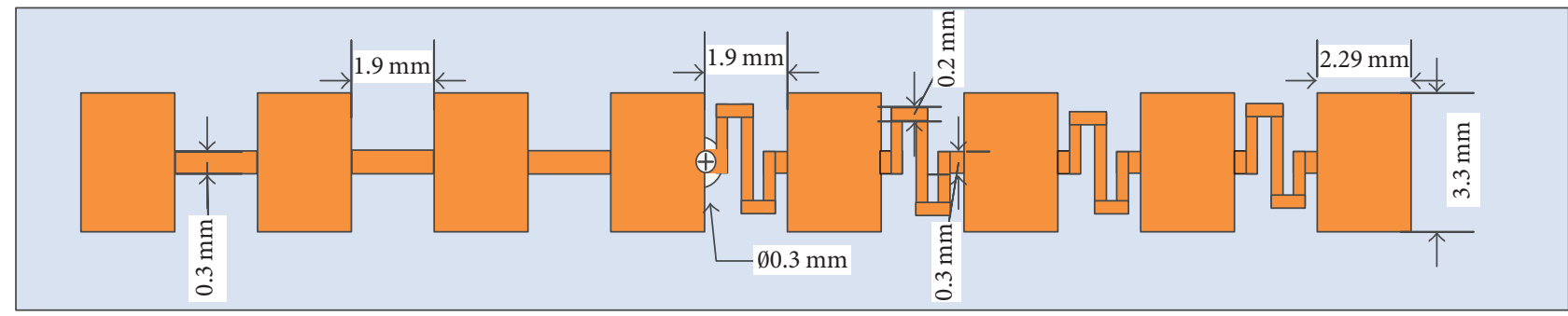

FIGURE 10: A uniformly distributed rectangular array with intermediate feeding.

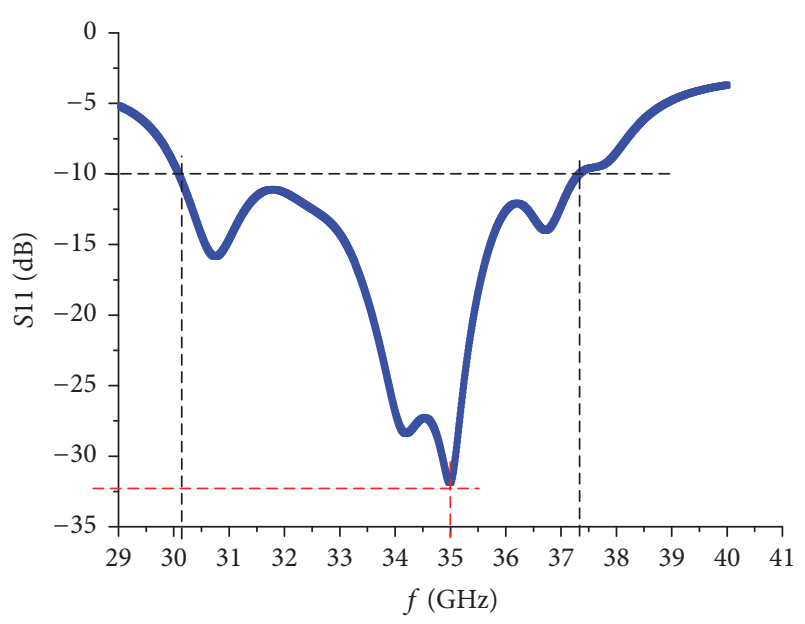

FIGURE 11: $S$-parameter of a rectangular array with uniform distribution with intermediate feeding.

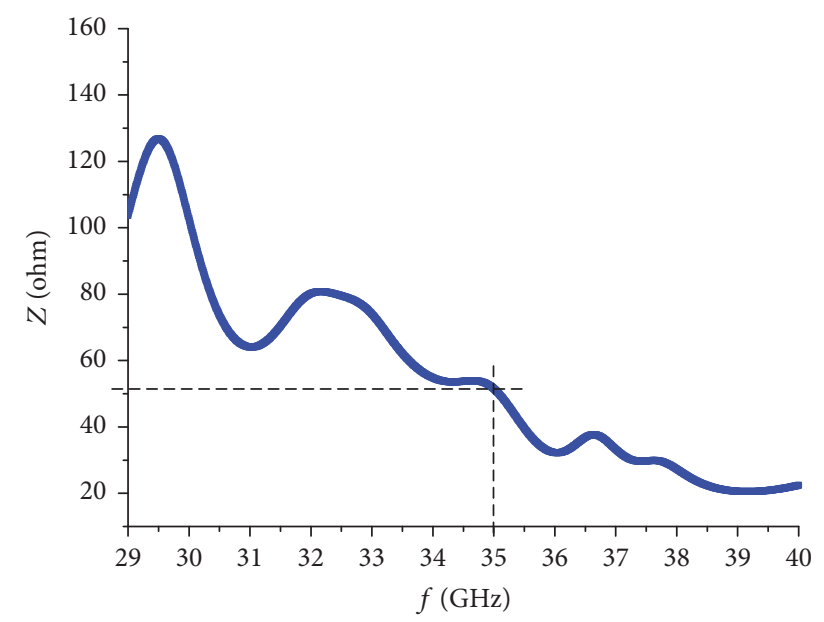

FIGURE 12: Impedance of a rectangular array with uniform distribution with intermediate feeding.

that the maximum gain is $13.9 \mathrm{~dB}$, the first sidelobe level is $-15.6 \mathrm{~dB}$, the main beam deflection offset is achieved on $E$ plane, the angle is about $60^{\circ}$, and the $3 \mathrm{~dB}$ lobe-width in the $E$-plane is $20^{\circ}$.

Three kinds of arrays are given in this design. The first two arrays actually provide reference for the Taylor distribution matrix. The first array provides an appropriate spacing of the

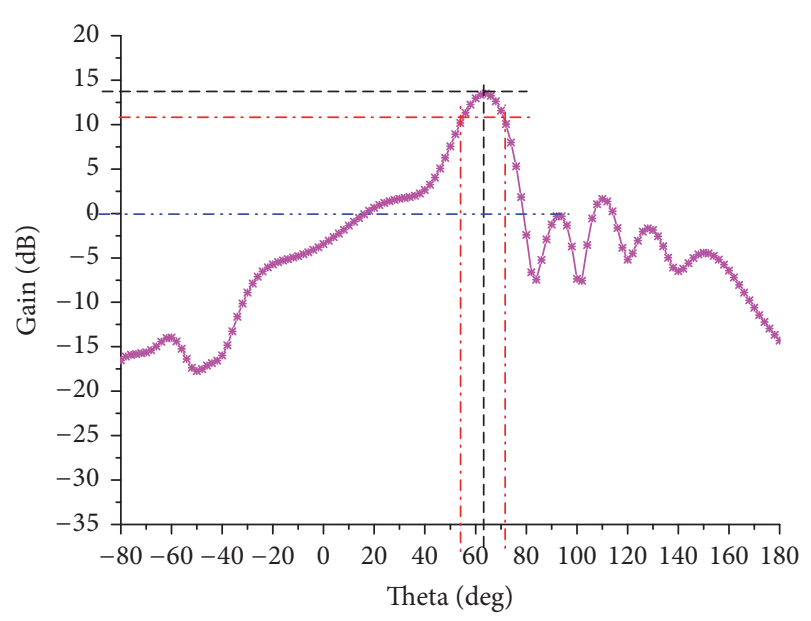

FIGURE 13: The gain of $E$-plane of a rectangular array with uniform distribution with intermediate feeding.

radiating elements. The second one determines the length of the serpentine. The final array form is based on the two arrays to adjust the radiation side of each radiating element to realize the current redistribution. In the array of rectangular patch, the gain is higher in the form of uniform distribution with intermediate feed. The lowest sidelobe level is the Taylor distribution with intermediate feed to reduce the sidelobe. The narrowest beam and the best matched impedance are the uniform distribution with one end of the feed. It can be seen that the reduction of the first sidelobe level is at the expense of width of the main lobe.

\section{Design of Conformal Arrays}

The conformal array is designed in the third part of the MIMO conformal antenna, and the conformal carrier is the cylinder with a diameter of $60 \mathrm{~mm}$ [23].

The center frequency of the design is $35 \mathrm{GHz}$, and the dielectric substrate with relative dielectric constant $\varepsilon_{r}=2.2$ is selected. Thickness of the substrate is $0.5 \mathrm{~mm}$. According to the design of the radiation unit, the size of the microstrip patch antenna is only about $3 \mathrm{~mm}$. The curvature of $60 \mathrm{~mm}$ cylindrical diameter is smaller than the microstrip patch antenna. So the antenna can be regarded as a planar antenna and analyzed by the theory of planar antenna. The design needs to achieve a specific beam direction, which is $60^{\circ}$ to the conformal vector axis, and can be realized by conformal 


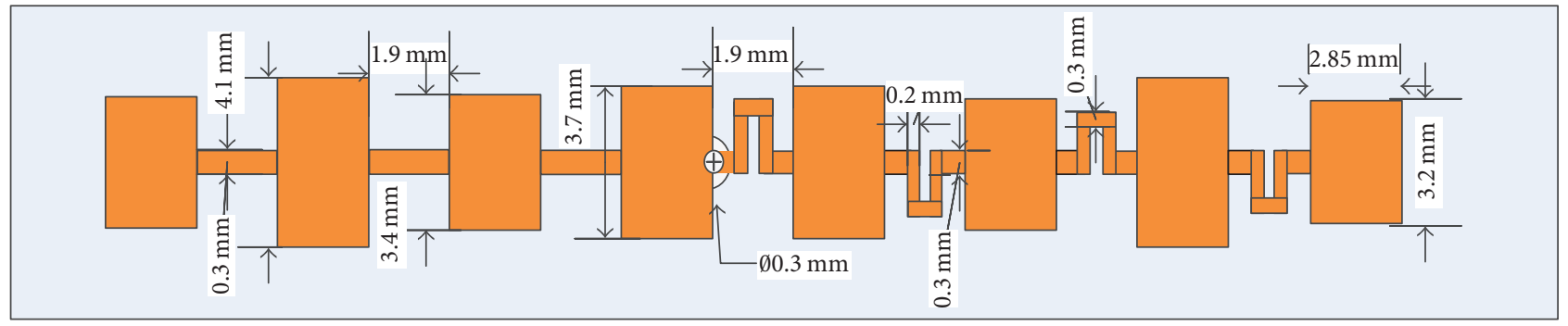

FIGURE 14: A rectangular array with Taylor distribution with intermediate feed.

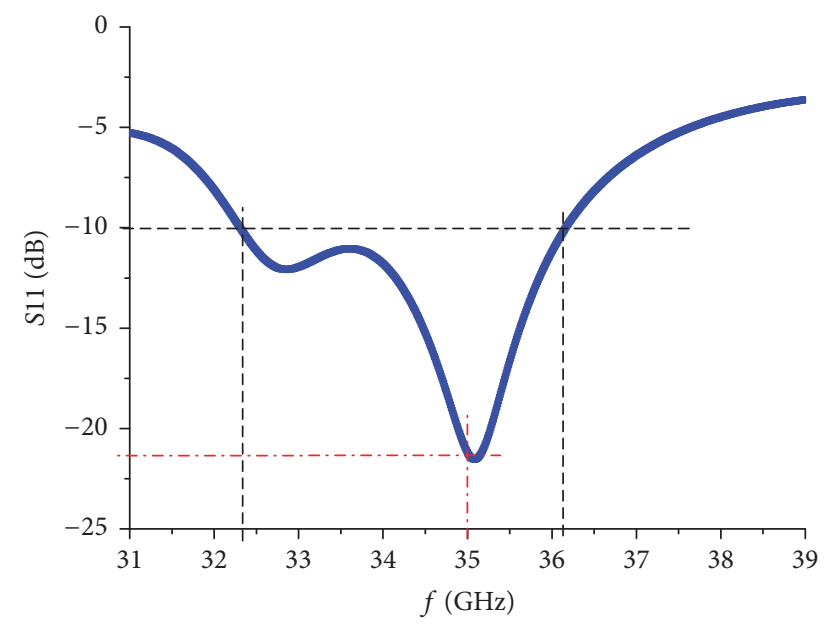

FIGURE 15: S-parameter of a rectangular array with Taylor distribution with intermediate feeding.

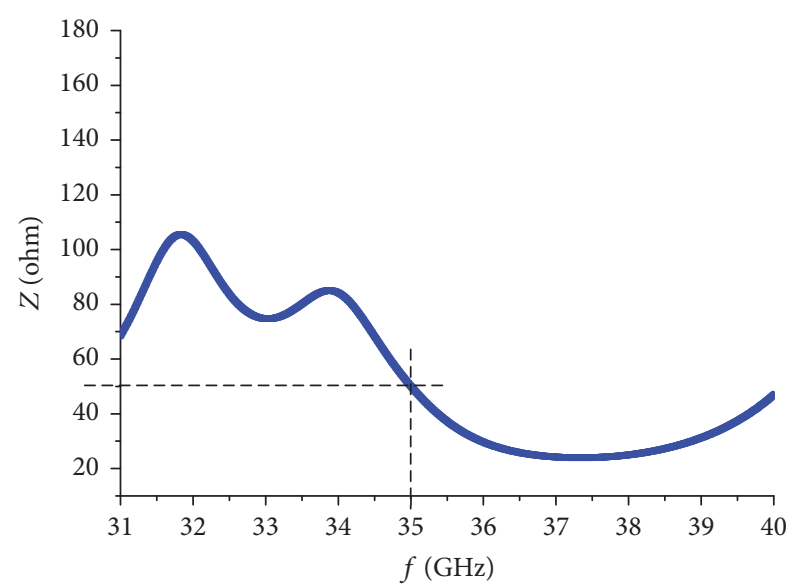

FIGURE 16: Impedance of a rectangular array with Taylor distribution with intermediate feeding.

array. According to the analysis of the series-feed array, the microstrip patch antenna can be composed of a series-feed array to achieve such a beam direction. It can be realized by adjusting the spacing between the elements. The low sidelobe can be realized by the Taylor synthesis method. The distribution current of the antenna array is tapered to reduce the sidelobe level.

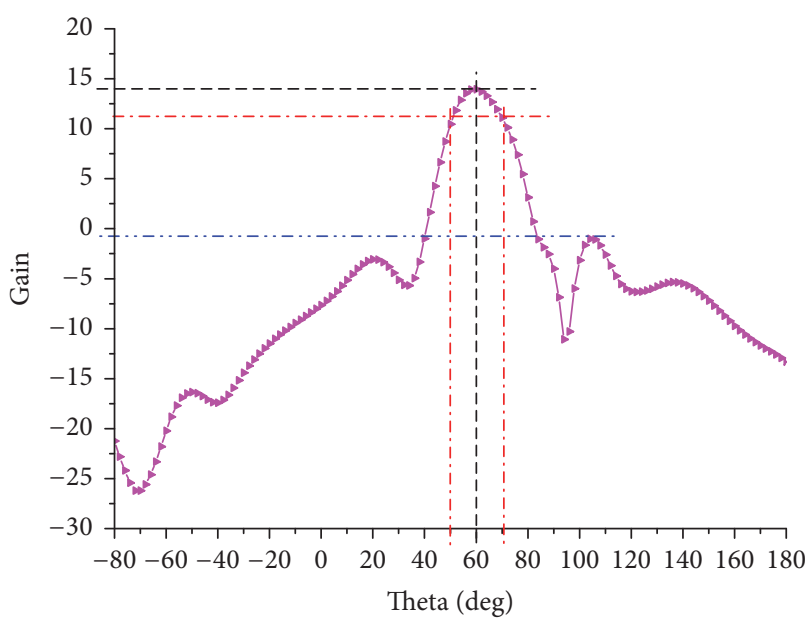

FIgURE 17: The gain of $E$-plane of a rectangular array with Taylor distribution with intermediate feeding.

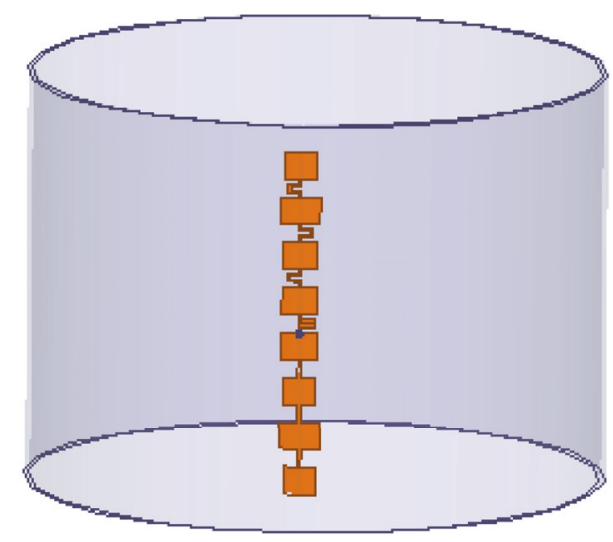

FIgURE 18: The microstrip antenna conformal array.

The microstrip antenna conformal array is shown in Figure 18.

Through simulation and optimization, the radiation characteristic of microstrip antenna array is obtained. The $S$ parameter is shown in Figure 19. The return loss of the conformal array is down to $14 \mathrm{~dB}$ at $35 \mathrm{GHz}$. The relative bandwidth of $\left|S_{11}\right|<-10 \mathrm{~dB}$ can be calculated to be $11.8 \%$.

The input impedance of the array is shown in Figure 20. It can be seen that the whole antenna is matched to $45 \Omega$. The 


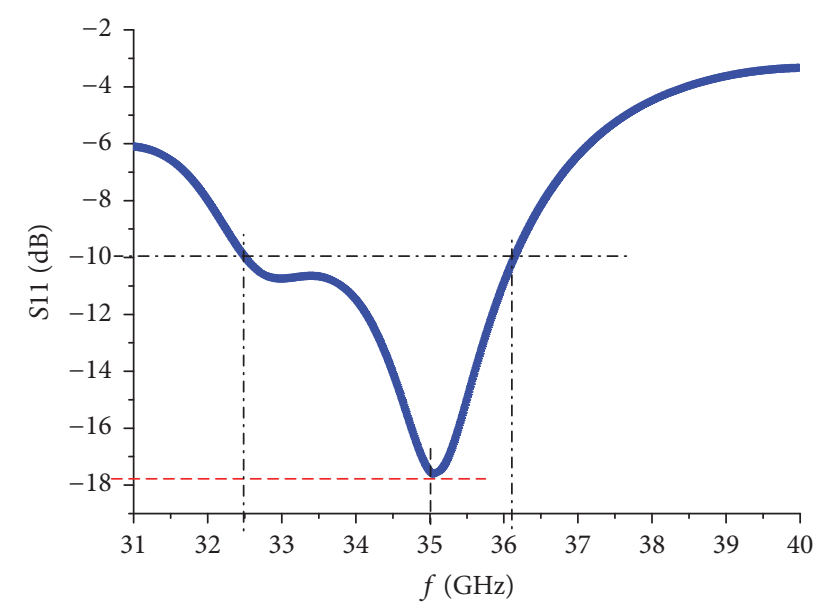

FIGURE 19: $S$-parameter of conformal microstrip antenna array.

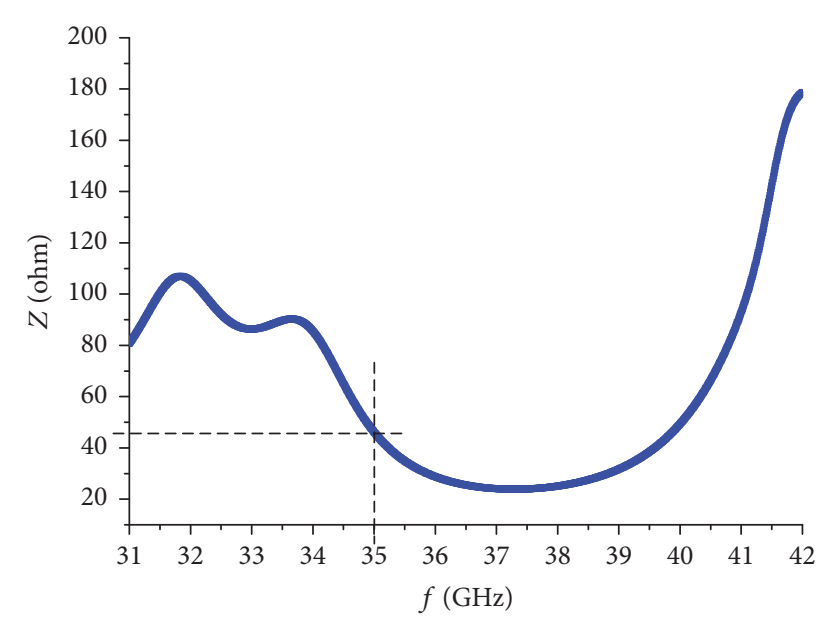

FIGURE 20: Input impedance of conformal microstrip antenna array.

gain of E-plane microstrip antenna conformal array is shown in Figure 21. It can be seen that the maximum gain of the array is $13.3 \mathrm{~dB}$, and the first sidelobe level is $-16 \mathrm{~dB}$. The $E$-plane realized the main beam offset. The offset angle is about $62^{\circ}$. The $3 \mathrm{~dB}$ beam width of $E$ plane is $18.2^{\circ}$.

We fabricated a pair of 8 -element series-fed conformal antenna array, as shown in Figure 22. The analysis and test results are compared.

$S$-parameters are shown in Figure 23. It can be seen from the results of the $S$-parameters of the antenna that the resonance point of the measurement and simulation is consistent. The measurement results of the antenna relative bandwidth is about $11 \%$, which is slightly less than the simulation results.

The comparison of measurement results and simulation results of normalized pattern of $E$ plane is shown in Figure 24. It can be seen that the radiation plot of $E$-plane achieves the main beam offset. The angle is about $62^{\circ}$ and this meets the requirements. The first sidelobe level rose to $-14 \mathrm{~dB}$. The $3 \mathrm{~dB}$ lobe-width of $E$-plane is about $17^{\circ}$. The measurement results are in good agreement with the simulation results.

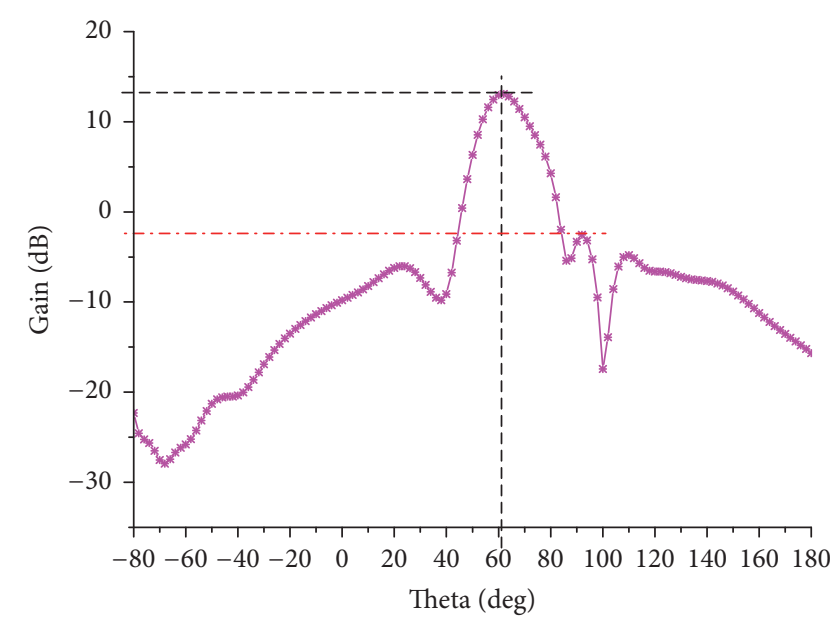

FIGURE 21: Gain of E-plane of conformal microstrip antenna array.

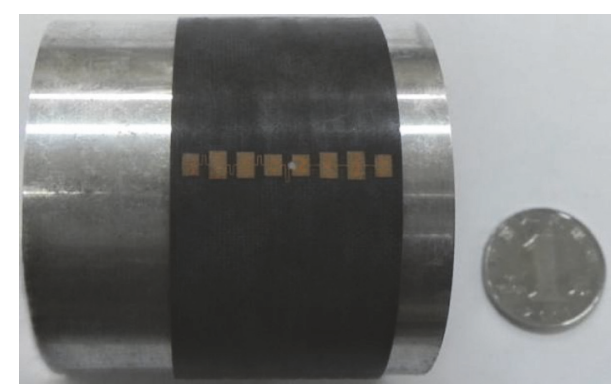

FIGURE 22: Eight-element series-fed conformal antenna array.

The gain measurement is performed by comparing to a standard horn antenna. The gain of the antenna is $12.2 \mathrm{~dB}$.

\section{Coupling Analysis of 5G MIMO Conformal Antenna}

It is also very important to decide the number of the antennas in the design of MIMO conformal antenna. Antenna coupling has significant impact on radiation pattern. When using multiple antennas, the cross coupling between the antennas should be discussed and the coupling needs to be minimized. The main factor that affects the coupling is the distance between antennas. The closer the distance between antennas, the stronger the coupling. Therefore, we should find the appropriate antenna spacing to meet the performance requirement of the antenna coupling. At the same time, this determines the maximum number of antennas [24]. For conformal system, the diameter of the conformal carrier is $60 \mathrm{~mm}$. The carrier space is limited, so the number of RF circuits is limited. At the same time, too many RF circuits will increase the cost of the system. Therefore, the number of antennas needs to be determined by considering these factors.

As we know, the energy of array antenna can be coupled by space wave or surface wave, when the coupling level is greater than $-20 \mathrm{~dB}$, the performance of the antenna will be greatly affected [25]. In this design, each antenna is used 


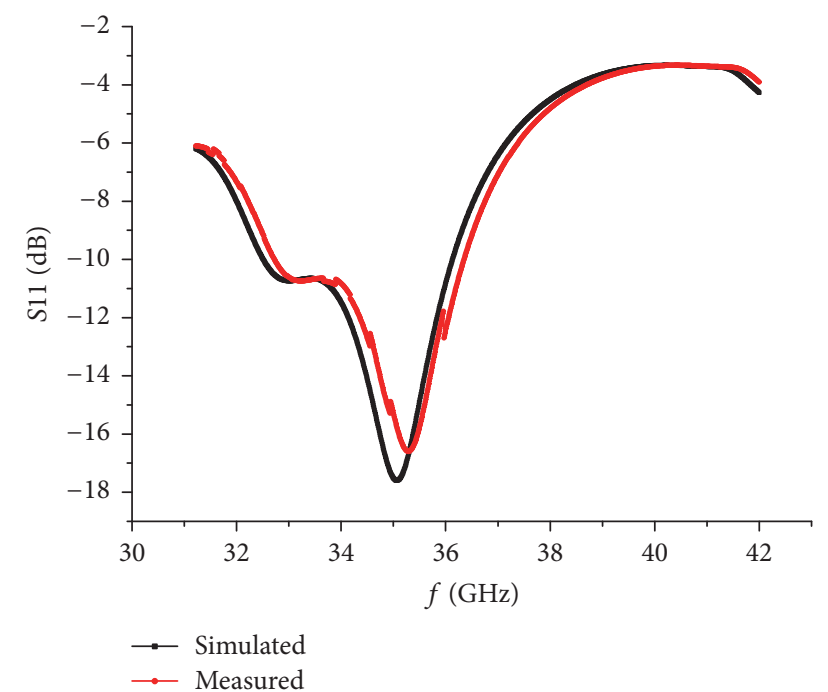

FIGURE 23: Comparison of $S$-parameters and simulation results of the 8-element series-feed conformal antenna array.

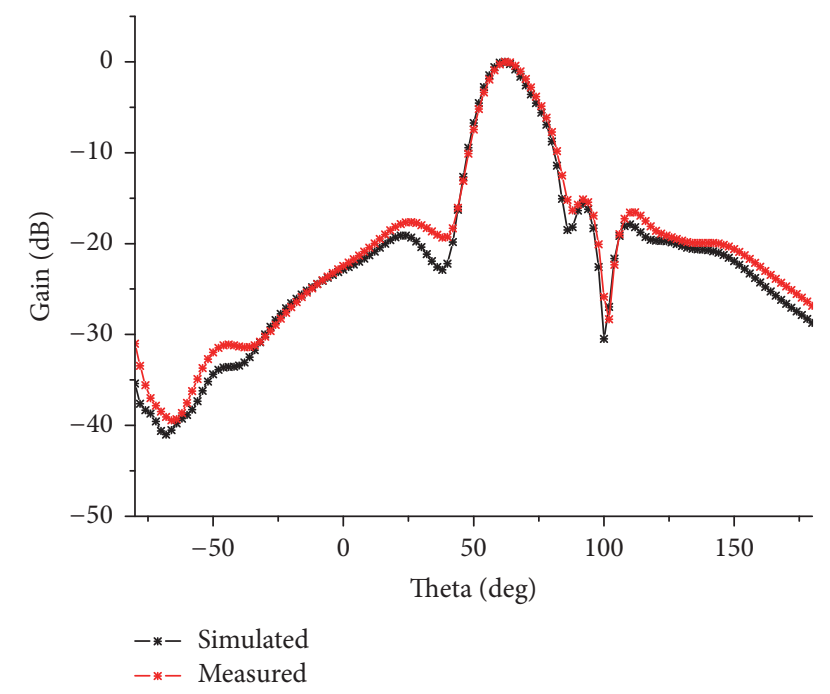

FIGURE 24: Comparison of the measurement and simulation results of the $E$ surface orientation of the 8-element series-fed conformal antenna array.

as an independent antenna, so the requirement of coupling between the antennas is low, and the coupling between antennas is $-40 \mathrm{~dB}$. Through simulation and optimization, we find that when the number of the antennas is eight, it can meet the requirement of the coupling of antenna to be less than $-40 \mathrm{~dB}$. Eight antenna arrays can be placed on the conformal carrier. Actually we cannot put so many antennas. First of all, to consider the cost of the RF circuit, eight arrays of antennas require eight RF links. Secondly, to consider the volume of the conformal carrier, not more than four radio frequency links can be placed in this limited space. Therefore this design uses four arrays. Combined with the spatial symmetry of the antenna, the four pairs of antennas are distributed with equal distance in the conformal cylindrical carrier. The simulation model is shown in Figure 25.

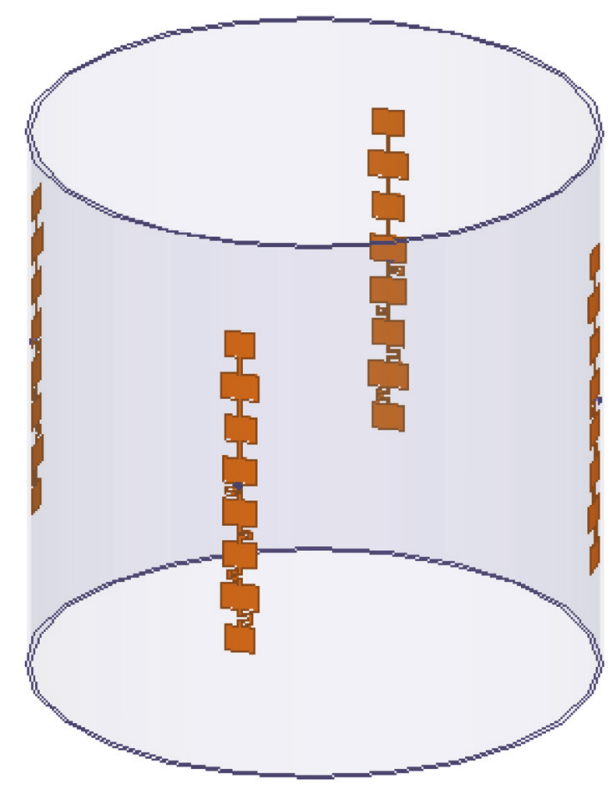

FIGURE 25: MIMO radar conformal antenna.

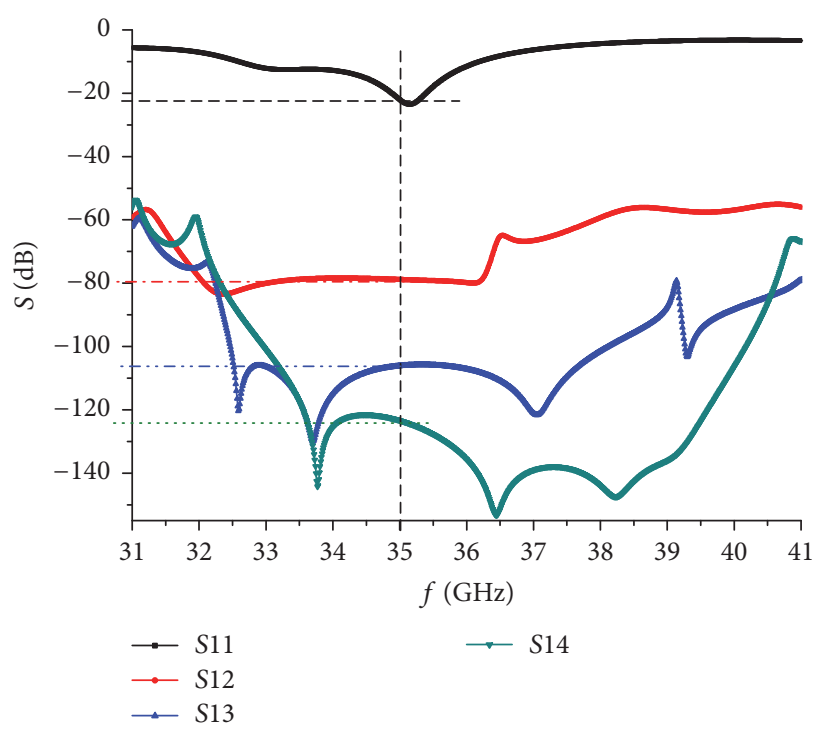

FIGURE 26: S-parameters of MIMO conformal antenna 1.

The $S$-parameters of the first antenna in the MIMO radar conformal antenna are shown in Figure 26. It can be seen from the figure that the antenna's reflection parameters are $-21.5 \mathrm{~dB}$. Considering the coupling of antennas, it is clear that the cross coupling satisfies the requirements of $-40 \mathrm{~dB}$.

The radiation plot of the first antenna in the MIMO radar conformal antenna is shown in Figure 27. It can be seen from the figure that, by considering the coupling effects, the first sidelobe level has been significantly improved to $-11.5 \mathrm{~dB}$. This is consistent with the theoretical analysis.

The fabricated conformal antenna with 4 arrays of antennas is shown in Figure 28. 


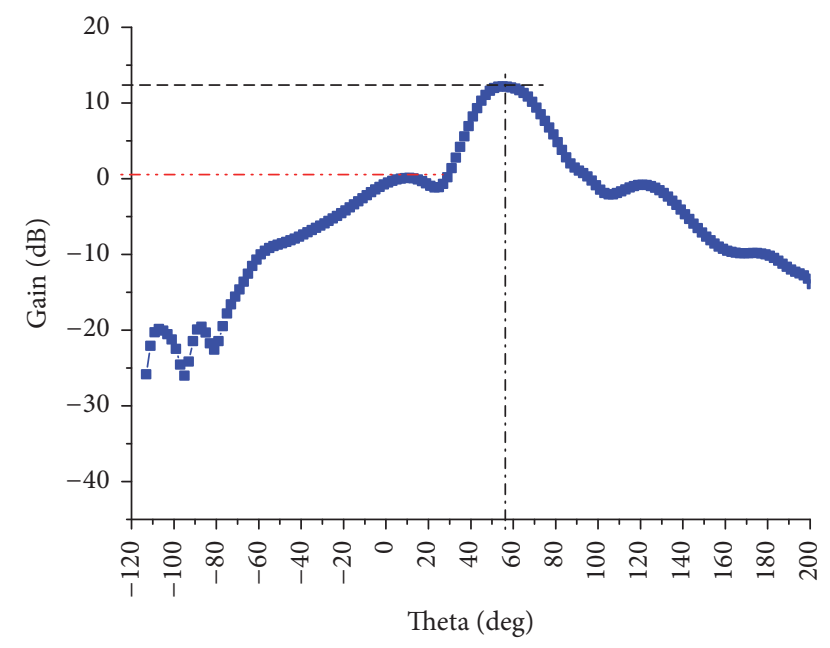

FIGURE 27: Gain of $E$-plane MIMO conformal antenna 1.

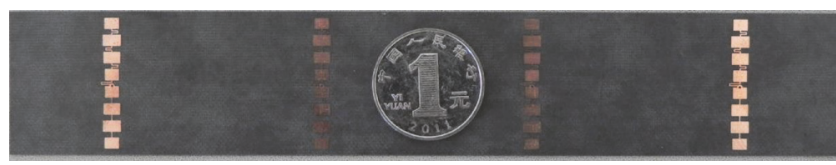

Figure 28: Planar expansion of four pairs conformal microstrip antenna.

\section{Conclusion}

In this paper, in order to meet the requirements of the antenna system, an 8-cell series-fed microstrip standing wave antenna array by traveling-wave theory has been designed in Ka band $(35 \mathrm{GHz})$. The deflection of the main lobe and the plane of array is realized by adjusting the spacing between the elements. At the same time, the Taylor distribution is used for the antenna synthesis, and the first sidelobe level is reduced by controlling the current amplitude of the unit. Next, MIMO conformal antenna at $35 \mathrm{GHz}$ is designed. The bandwidth of antenna is greater than $10 \%$, the gain is greater than $10 \mathrm{~dB}$, and the first sidelobe level is reduced to $-16 \mathrm{~dB}$. The angle between the main lobe and the carrier axis is $60^{\circ}$. The measurement results agree well with the simulation results, which meet the requirements of the system to the antenna performance. Considering the cost of system, space limitation, and antenna coupling, we design four 8-cell series-fed microstrip standing wave antenna arrays. The four antenna arrays are evenly distributed on the conformal of carrier, and the cross coupling of the antenna is lower than $-40 \mathrm{~dB}$.

\section{Conflicts of Interest}

The authors declare that they have no conflicts of interest.

\section{References}

[1] YaoHuan Gong, "Multiple input multiple out of smart antenna technology," ZTE Technology, vol. 6, pp. 19-21, 2002.

[2] Li Gang Ren and Mei Song, "MIMO technology in mobile communication [J]," Modern telecommunication technology, vol. 1, pp. 42-45, 2004.
[3] G. J. Foschini, "Layered space-time architecutre of wireless communication in a fding environment when using multielement antennas," Bell Labs Technical Journal, vol. 1, no. 2, pp. 41-59, 1996.

[4] G. J. Foschini, "On limits of wireless communications in a fading environment when using multiple antennas," Wireless Personal Communications, vol. 6, no. 3, pp. 311-335, 1998.

[5] WeiHong Xiao, Multi Antenna Design for MIMO Mobile Communication System, Xidian University, Xi'an, China, 2006.

[6] YanJie Zhang, Research on Coupling Characteristics of MIMO Antena, Xidian University, Xi'an, China, 2012.

[7] Z. Yang, H. Yang, and H. Cui, "A compact MIMO antenna with inverted C-shaped ground branches for mobile terminals," International Journal of Antennas and Propagation, vol. 2016, Article ID 3080563, 2016.

[8] Kyungjung Kim., Sarkar, M. C. Wicks et al., "DOA Estimation Utilizing Directive Elements on a Conformal Surface," in Radar Conference, 2003. Proceeding of the 2003 IEEE, pp. 91-96, Huntsville, Alabama, 2003.

[9] R. K. Hersey, W. L. Melvin, and J. H. McClellan, "Clutter-limited detection performance of multi-channel conformal arrays," Signal Processing, vol. 84, no. 9, pp. 1481-1500, 2004.

[10] Z. Li, X. Kang, J. Su, Q. Guo, Y. L. Yang, and J. Wang, “Clutter Limited Detection Performance of Multi-channel Conformal Arrays," International Journal of Antennas and Propagation, vol. 2016, Article ID 9812642, 2016.

[11] T. E. Morton and K. M. Pasala, "Pattern synthesis of conformal arrays for airborne vehicles," in Proceedings of the 2004 IEEE Aerospace Conference Proceedings, pp. 1030-1038, Big Sky, Montana, USA, March 2004.

[12] R. K. Mishra and A. Patnaik, "Design of circular microstrip antanna using neural networks," IETE Journal of Research, vol. 44, no. 1-2, pp. 35-39, 2015.

[13] A. Sayed, R. Ghonam, and A. Zekry, "Design of a Compact Dual Band Microstrip Antenna for Ku-Band Applications," International Journal of Computer Applications, vol. 115, no. 13, pp. 699702, 2015.

[14] DongLiang Zhao, "Thoughts on the development of 5G mobile communication," Information Communication, vol. 9, 2015.

[15] HongJie Yao, "The key technology and process of 5G mobile communication," Communication World, vol. 6, 2015.

[16] Warren L.Stutzman and Gary A. thiele, Encyclopedia of RF and Microwave Engineering, People's Posts and Telecommunications Publishing House, China, 2nd edition, 2006.

[17] YuFang Tang, Theoretical study and engineering application of microstrip line loss, Nanjing University of Science and Technology, 2009.

[18] Jun zhang and KeCheng Liu, Microstrip antenna theory and Engineering, National Defense Industry Press, China, 1988.

[19] ShuJie Li, Research on Microstrip Planar Array Antenna in Ku Band, Xidian University, Xi'an, China, 2006.

[20] DaJun Wu, Design And Research of Millimeter Wave Cylindrical Conformal Microstrip Antenna, Nanjing University of Science and Technology, Nanjing, China, 2007.

[21] XingJian Kang, Principle And Design of Antenna, National Defense Industry Press, China, 1995.

[22] ChuFang Xie, Modern Antenna Theory, Chengdu Telecommunication Engineering Institute Press, Sichuan, China, 1987.

[23] Song Zhu, "Development of conformal antenna and its application in electronic warfare," Journal of the Chinese Academy of Electronic Science, vol. 12, 2007. 
[24] ChangSheng Shi, "Isolation of antenna," Electronic Science and Technology Revie, vol. 11, pp. 16-18, 1997.

[25] QiaoLong Lan, Research on Millimeter Wave Microstrip Antenna, University of Electronic Science and technology, Chengdu, China, 2006. 


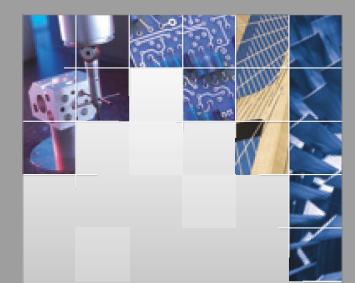

\section{Enfincering}
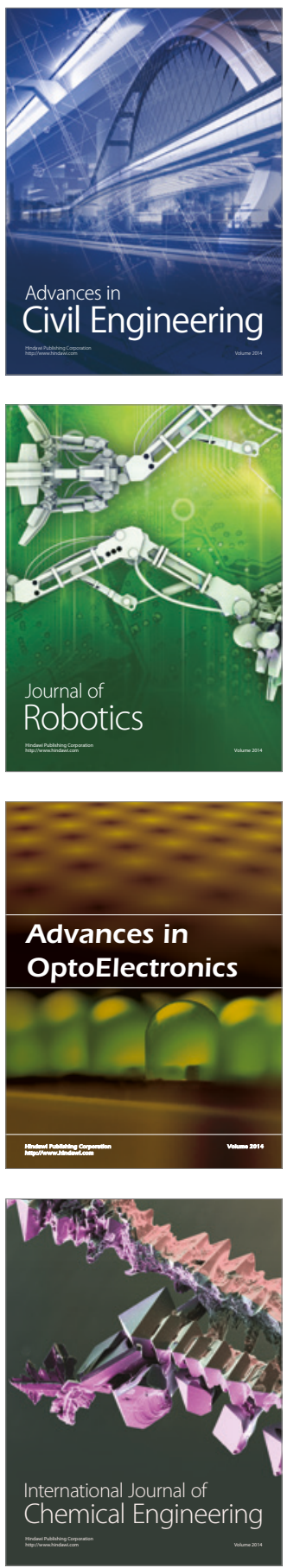

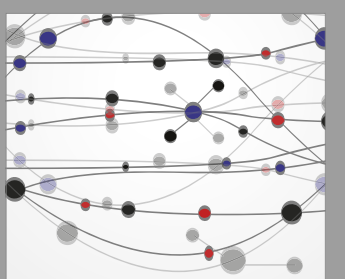

The Scientific World Journal

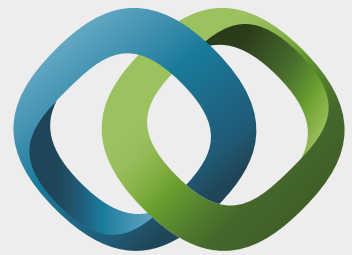

\section{Hindawi}

Submit your manuscripts at

https://www.hindawi.com
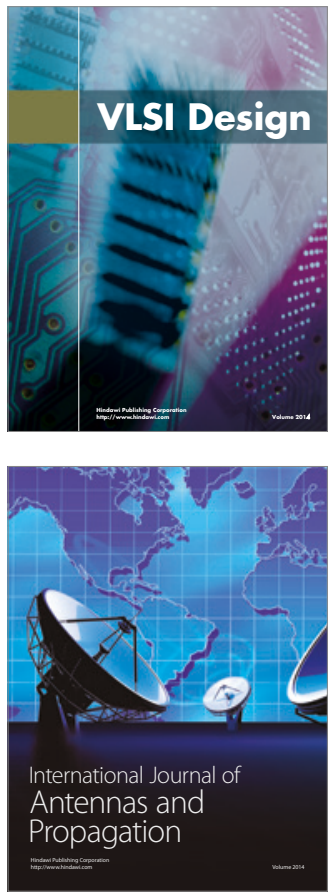

\section{Rotating}

Machinery
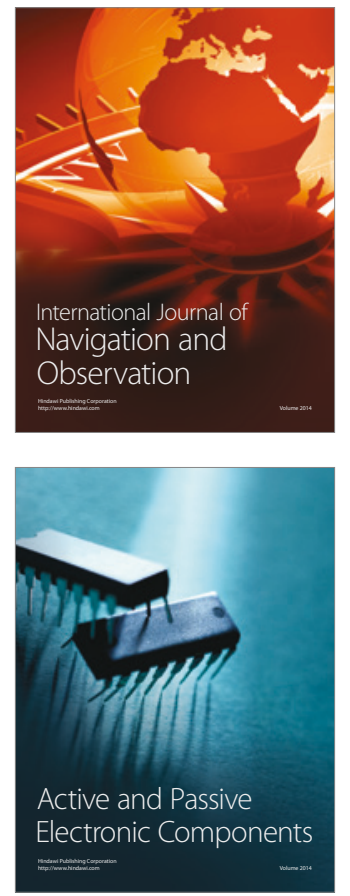
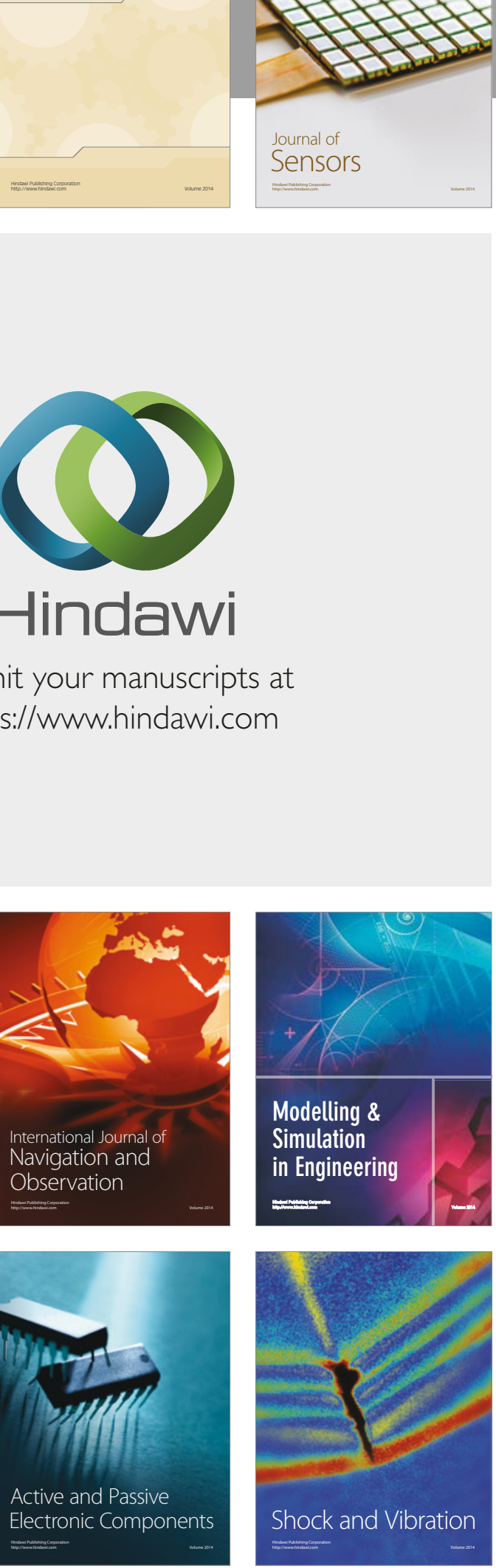
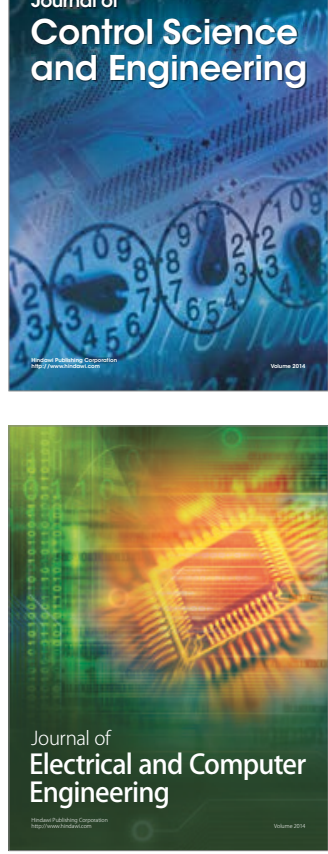

Distributed

Journal of

Control Science

and Engineering
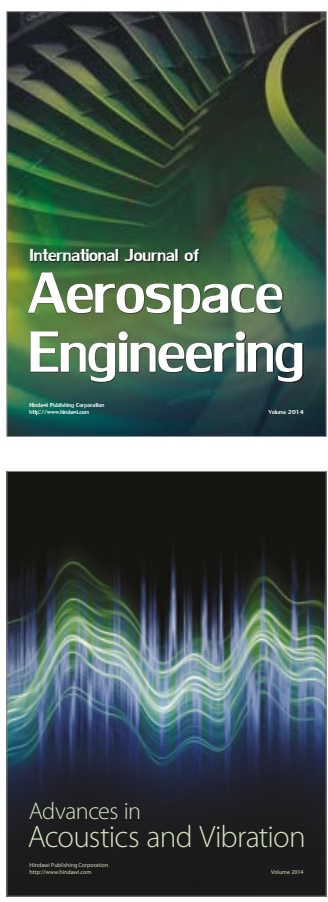

Sensor Networks 